\title{
The speed of visual processing in children and adults: Effects of backward and forward masking ${ }^{1}$
}

\author{
PHILLIP H. LISS, 2 CITY COLLEGE OF THE CITY UNIVERSITY OF NEW YORK \\ AND MARSHALL M. HAITH, HAR VARD UNIVERSITY
}

Children, $4-5$ and 9-10 years old, and college-age adults were tested on two visual masking tasks. The first task required the Ss to identify whether a tachistoscopically presented bar was horizontal or vertical. The second task, also using tachistoscopic presentation of stimuli, required $S s$ to locate a horizontal (or vertical) bar in a matrix of vertical (or horizontal) bars. In both tasks, backward masking produced greater disruption than did forward masking, and the amount of disruption induced by both backward and forward masking decreased as age increased. An Age by Masking Condition interaction was found only in the location task and reflected a much greater difference between backward- and forward-masking conditions for the youngest group than for the older groups. On the basis of these findings and other considerations, it was concluded that only in the location task, which presumably required visual search, was the speed of visual processing slower in the younger group.

A recent study compared preschool children and college adults in the recognition of single outlined geometric forms presented tachistoscopically (Haith et al, 1969). The children's thresholds were about $8 \mathrm{msec}$ greater than the adults'. This difference was tentatively interpreted not in terms of differences in sensory sensitivity but rather in terms of the superior employment by adults of partial cues as the threshold exposure duration was approached.

As in most tachistoscopic experiments, the previous study primarily measured the sensitivity of the visual system to very weak stimuli. Since the visual persistence of a stimulus outlasts its actual physical exposure (Sperling, 1960), no direct measure of the speed of visual processing was possible. Thus, the finding of similar duration thresholds in preschoolers and adults did not necessarily reflect similar

Fig. 1. Graph showing an example of a stimulus card (TS) and the masking card (MS) used in Experiment 2. The edges of the inscribed crosses in the MS were coincident with the edges of the relevant bars in the TS. (Diagram after Averbach \& Coriell, 1961.) visual-processing times. In fact, it is possible that larger age differences were disguised by a longer visual persistence in children.

Suppose, after presenting a target stimulus (TS) that must be identified, a second stimulus, a backward mask (BM), is presented that covers the relevant features of the visual trace of TS. Several lines of evidence suggest that the visual system is then unable to analyze the TS for any time longer than the time between the onset of the TS and the onset of the mask (e.g.; Liss, 1968; Sperling, 1963). Therefore, it is suggested that backward masking can be used to investigate the time required to process a stimulus. The studies reported here assume this evidence to be valid and employ backward masking to control visual processing time.

Since age differences in a backward-masking situation might reflect differences in motivation, task comprehension, or usage of subtle cues, as well as visual-processing speed, we also used a forward-masking (FM) procedure in which the mask preceded the TS as a control. It was assumed that age differences unrelated to processing time would be similar in the FM and BM conditions but that processing time would be unaffected by the mask in the FM condition. By this line of reasoning, age differences found with backward masking, but not with forward masking, could be attributed to differences in processing speed.

Two experiments were performed. The stimuli and procedures in the first experiment were chosen to minimize the effects of memory load and of age differences in the difficulty of reporting the stimuli. The TS was a single line, and S simply had to report whether the line was horizontal or vertical. In the second experiment, the task was slightly more complex. The TS was one line that had a horizontal (or vertical) orientation but was a component of a 3 by 3 matrix of lines; the eight non-TS lines were in the opposite orientation and S's task was to identify the location of the TS line. Thus, visual processing time was assessed for various age groups in both a simple identification task and in a more complex visual-search task.

\section{METHOD}

Subjects

Eight Ss from each of three age groups-4-5 years, 9-10 years, and adults-participated in the experiment. The children were seen in an elementary school, the adults in William James Hall at Harvard University.

\section{Equipment and Materials}

A Gerbrands three-field mirror

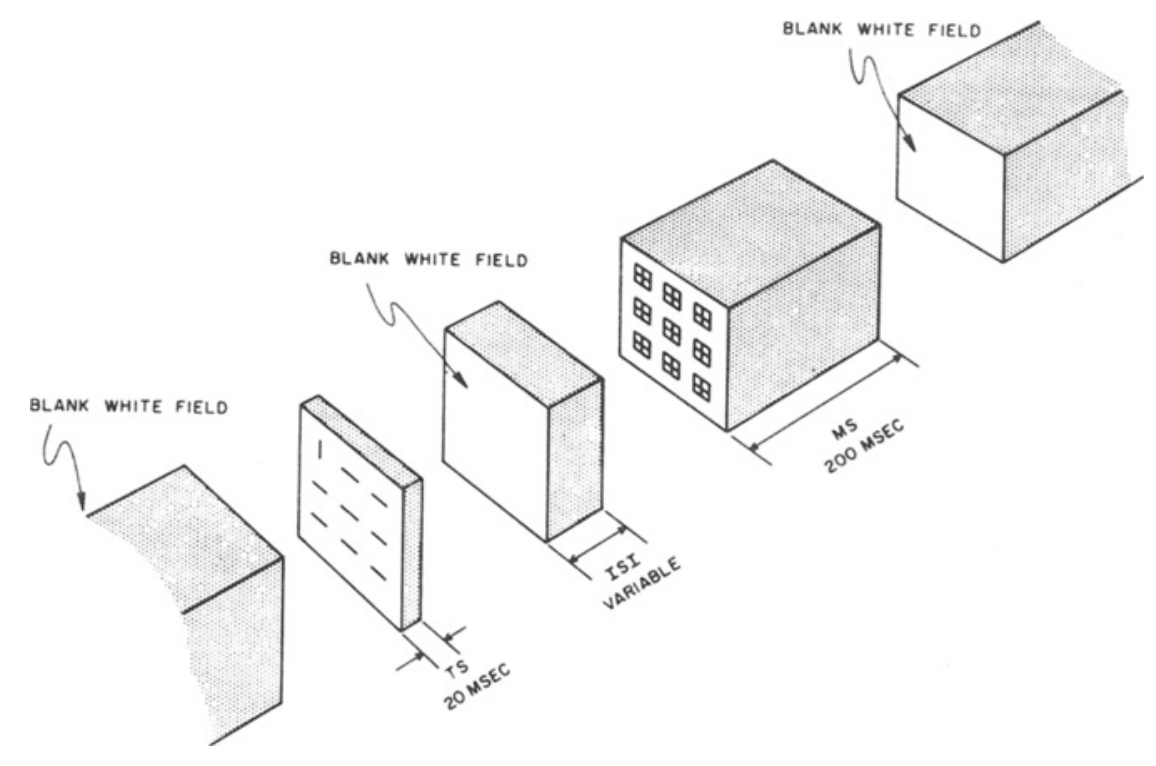




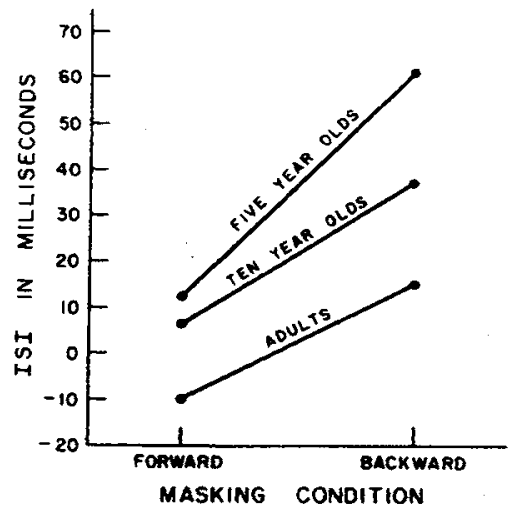

Fig. 2. Mean interstimulus interval (ISI) at which threshold criterion was reached as a function of age and masking condition in Experiment 1.

tachistoscope ( $\mathrm{T}$-scope) was used in conjunction with two solid-state timers that were adjustable from $10 \mathrm{msec}$ to $1,000 \mathrm{msec}$ in 10 -msec steps $(2 \%$ accuracy). Each stimulus field was illuminated by two 4-W fluorescent tubes viewed through two Liberty 30-30 mirrors (Libbey-Owens-Ford glass, Spec. No. 1064). A button, mounted in a hand-held box and activated by $S$, served to trigger a timed sequence in which the TS and mask were presented. The fields of the $T$-scope were $18.4 \times 18.4 \mathrm{~cm}$, thus providing a white $12-\mathrm{deg} 46-\mathrm{min}$ square viewing area. Field illumination was approximately $1.9 \mathrm{fL}$ for all fields.

Two sets of stimulus cards (Chart-Pak) were prepared by total transfer of individual black solid bars, $1 / 2 \times 1 / 8 \mathrm{in}$., on to white bristol-board cards. For Experiment 1, target cards were prepared that contained a single centered bar in either a horizontal or a vertical orientation. (At the viewing distance of 32 in., the bars were $53 \mathrm{~min}$ long by $13 \mathrm{~min}$ wide.) The MS was prepared from six similar bars arranged to form a centered black cross surrounded by a black square. The outside edges of the cross bars coincided with the inside edges of the square.

For Experiment 2, each stimulus card contained a 3 by 3 matrix of horizontal and vertical bars. Eighteen cards were prepared in which the TS, horizontal or vertical, was in each of the nine possible spatial positions; the remaining eight bars were in the opposite orientation. The visual angle from the center of the array to the center of the corner bars was $2.5 \mathrm{deg}$. A masking card contained nine crosses inscribed in nine squares, which were centered in each of the nine stimulus positions (see Fig. 1). A white fixation card illuminated before a trial and during the TS-MS interval contained only a tiny black fixation dot in the center.

\section{Procedure}

Experiment 1. The S looked at the tiny dot on the fixation card and pressed a button, which initiated a trial. Several warm-up trials were first given each $S$ in which the TS was presented alone. When the $\mathrm{E}$ was satisfied that $\mathrm{S}$ understood the task, the experimental phase was begun. The button press was followed by a delay of $750 \mathrm{msec}$, in which the fixation field was maintained, and then a sequence of flashes. For backward masking, the sequence consisted of: (1) target for $20 \mathrm{msec}$, (2) fixation field for a variable interval (ISI), (3) mask for $200 \mathrm{msec}$, (4) fixation field. The temporal parameters were identical for forward masking except that (1) and (3) were reversed in sequence. The centers of the fixation point, the TS, and the mask were exactly superimposed and checked before each session. S reported the orientation of TS by pointing to a vertical or horizontal bar on a card to the right of the $T$-scope viewing mask. Feedback on performance after each trial was given all $\mathrm{Ss}$; in addition, for the youngest Ss, E added a chip to a stack of chips on top of the $T$-scope for each correct trial.

The FM and BM conditions were presented, in counterbalanced order, in a single session, separated by a 5 -min break. In the BM condition, after four successive correct trials at an ISI of $300 \mathrm{msec}$, a descending order of ISIs-150, 110,80, 50, 30,10 , and $0 \mathrm{msec}$-was initiated. If the $S$ responded correctly on the first four trials at a given ISI he was then presented trials at the next lowest ISI. If one or more errors were made, the $S$ was given eight trials at the same ISI before he was presented the next ISI. This procedure continued until a threshold criterion of three incorrect trials out of eight was reached. ${ }^{3}$ The FM condition was identical except that the ISI of $150 \mathrm{msec}$ was not used.

Experiment 2. Approximately 1 week after Experiment 1, the same $S$ s were tested in Experiment 2. The nine-bar stimulus cards were presented in randomized order under the same procedural conditions as in Experiment 1, with the exception that the threshold criterion was five errors instead of three errors out of eight trials. Ss reported the location of the TS by pointing to the corresponding position on a nine-dot response card. For a particular $S$ the TS was always either vertical or horizontal.

\section{RESULTS}

The results of Experiment 1 are shown in Fig. 2. Analysis of variance of threshold ISI as a function of age and masking condition revealed a stable relationship

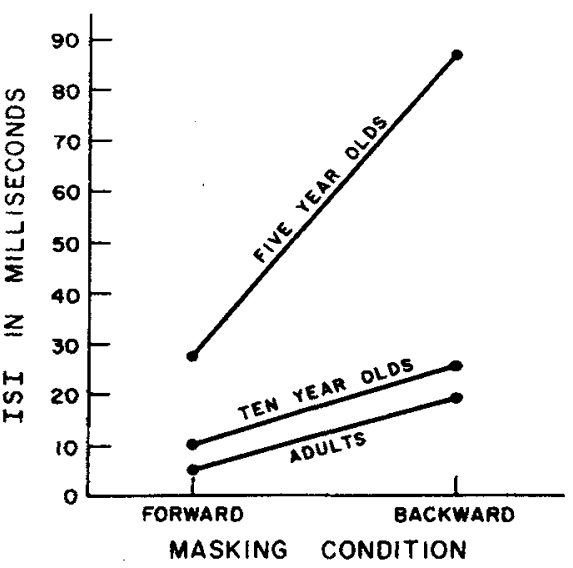

Fig. 3. Mean interstimulus interval (ISI) at which threshold criterion was reached as a function of age and masking condition in Experiment 2.

between age and threshold $(p<.001)$ and a stable effect of masking condition $(p<.001)$. The threshold decreased as age increased, and the threshold was higher for backward than for forward masking. The interaction between age and masking was not reliable.

The results of Experiment 2 are shown in Fig. 3. Analysis of variance revealed stable age and masking effects (both $p<.001$ ). As in Experiment 1, the thresholds decreased as age increased and were greater with backward masking than with forward masking. In contrast to Experiment 1, the interaction between age and masking was significant $(p<.01) .^{4}$

\section{DISCUSSION}

In both experiments, backward masking produced a greater decrement in performance than forward masking. The same result was found in a related study by Liss and Bower (1969). In most previous studies in which there was at least some superimposition of the contours of the target and mask, forward masking produced the greater decrement (Kahneman, 1968; Weisstein, 1968). The reason for this discrepancy in results is not known to us.

The age differences found in Experiment 1 were probably not due to differences in processing speed since they were about as great with forward as with backward masking. The obtained differences were probably due to the superior ability of adults in the use of subtle and partial cues. Contour summation may have provided one such cue, since some adult $\mathrm{Ss}$ reported that in near-threshold conditions they did not see the target but reported on the basis of which line of the mask appeared darkest. This factor may explain the ability of all 
adult Ss to perform above threshold at the 0 ISI interval with forward masking. 5

In Experiment 2, the significant Age by Masking Condition interaction indicates an age difference in processing speed, since age differences in the use of partial cues or in nonvisual factors were presumably identical for the FM and BM conditions. This difference in processing speed might reflect either a difference in processing strategy-serial processing by younger children and parallel processing by older children and adults - or in the rate at which the same strategy was implemented.

Surprisingly, for the two older groups, the backward-masking threshold was no greater in Experiment 2 than in Experiment $1 .^{6}$ Practice may have contributed to this effect. Two of the 9-year-olds were retested in the BM condition of Experiment 1 after finishing Experiment 2; the BM threshold of each was $20 \mathrm{msec}$ less than that first obtained. However, practice cannot be the sole explanation for these low thresholds in Experiment 2 because the BM threshold for the youngest group and the FM thresholds for all groups were greater in Experiment 2 than in Experiment 1. The similarity in BM threshold in the two experiments for the two older groups may, in fact, indicate that their speed of processing the nine bars in Experiment 2 was about the same as their speed of processing the single fixated bar in Experiment 1; i.e., the two older groups utilized a fast parallel processing strategy in Experiment 2.

A somewhat different interpretation of why the 10-year-olds and adults were so fast in processing the nine bars is that in Experiment 2, but not in Experiment 1, Ss knew beforehand the orientation of the TS; possibly, they "set" themselves for, say, a vertical bar. It may be argued that this set speeds processing and that 5-year-olds either could not use the knowledge of TS orientation to set a "preattentive" mechanism (Neisser, 1966) or that this strategy did not occur to them. One way to prevent the use of such a set, whatever its specific character, would be to have the orientation of the odd bar unpredictable from trial to trial. If the differences between the ages in processing speed were due to differences in the use of the proposed set, this change in procedure would eliminate the Age by Mask interaction. If, on the other hand, age differences in processing speed simply increase as task complexity increases, the proposed experiment would result in an even greater Age by Mask interaction.

It is intriguing to speculate on the possibility that FM and BM interfere with visual processing at different sites in the visual system. $\mathbf{A}$ number of studies have found that when the TS is presented to one eye and the MS to the other eye the interfering effect of the mask is more severely reduced for forward than for backward masking, in comparison to the monocular condition (see Kahneman, 1968 , for a general review).

If valid, this finding suggests that FM operates peripherally before interaction between impulses arising from the two eyes, whereas backward masking occurs more centrally. Following this argument, and coupling it with the present observations that the difference between FM and BM performance was very pronounced only for the 5-year-olds in the search task, it seems possible that the Age by Mask interaction represents adequate visual functioning of 5-year-olds at the peripheral level but not at a more central level. Unfortunately, the literature is not in total agreement regarding different dichoptic masking relations for BM and FM; Kinsbourne and Warrington (1962) found dichoptic masking to be as pronounced as monoptic masking for both forward and backward conditions. Accordingly, conclusions about the Age by Mask interaction as representing differences in central and not in peripheral processing for different age groups, although of interest, are somewhat premature.

\section{REFERENCES}

AVERBACH, E., \& CORIELL, A. S. Short-term memory in vision. Bell System Technical Journal, 1961, 40, 309-328.

HAITH, M. M., MORRISON, F. J., SHEINGOLD, K., \& MINDES, P. Short-term memory for visual information in children and adults. Paper presented at the annual meeting of the Eastern Psychological Association, Washington, D.C., April 1968.

KAHNEMAN, D. Method, findings and theory in studies of visual masking. Psychological Bulletin, 1968, 70, 404-425.

KINSBOURNE, M., \& WARRINGTON, E. K. The effect of an after-coming random pattern on the perception of brief visual stimuli. Quarterly Journal of Experimental Psychology, 1962, 14, 223-234.

LISS, P. Does backward masking by visual noise stop stimulus processing? Perception \& Psychophysics, 1968, 4, 328-330.

LISS, P., \& BOWER, T. G. R. Stages of visual processing: Evidence from backward masking and reaction time. Presented at Eastern Psychological Association meetings, Philadelphia, April 1969.

NEISSER, U. Cognitive psychology. New York: Appleton-Century-Crofts, 1967.

SPERLING, G. The information available in brief visual presentations. Psychological Monograph, 1960, 74, 11 (Whole No. 498).

SPERLING, G. A model for visual memory tasks. Human Factors, 1963, 5, 19-31.

WEISSTEIN, N. A Rashevsky-L andahl neural set: Simulation of metacontrast. Psychological Review, 1968, 75, 494-521. NOTES

1. The authors would like to thank Mr. Groblewski, the teachers and the staff at the Park School in Brookline, Mass., for their cooperation in providing space and subjects for this experiment. This investigation was supported by NICHD Grant HD-02680 to Marshall Haith.

2. Address: Department of Psychology, City College of the City University of New York, New York, New York 10031.

3. Ss were given an arbitrary threshold score of $-10 \mathrm{msec}$ if they did not reach a threshold criterion by the 0 ISI condition.

4. The relative difficulty of the BM condition in Experiment 2 was reflected by the number of errors made to our criterion of four successive correct at $300 \mathrm{msec}$. The median number of errors for the youngest, middle, and oldest groups, respectively, were $4.5,0$, and 0 , whereas for Experiment 1, the medians were 0 for all groups. That these differences cannot be dismissed as confusion or misunderstanding by the youngest group in Experiment 2 is illustrated by the observations that order of presentation of tasks had no effect on this measure, and that a median of only 1.0 errors was found for the youngest group under forward masking.

5. However, factors other than contour summation were involved in this perceptual task because, except for adults in the FM condition, Ss gradually dropped in accuracy as ISI decreased rather than improving in accuracy as contour-summation theory would predict.

6. Even though different threshold criteria were used for these two tasks, the chances of staying above threshold by guessing were somewhat less in Experiment 2 than in Experiment 1. The probability of a correct guess on any one trial was 1/2 in Experiment 1 and 1/9 in Experiment 2.

(Accepted for publication February 16, 1970.) 